Gut, 1972, 13, 21-23

\title{
Alterations in serum immunoglobulins after resection for ulcerative and granulomatous disease of the intestine
}

\author{
IRWIN M. GELERNT, DANIEL H. PRESENT, AND H. D. JANOWITZ \\ From the Department of Surgery and the Division of Gastroenterology, Department of Medicine, The Mount \\ Sinai School of Medicine of the City University of New York
}

SUMMARY Following resections for ulcerative colitis and granulomatous enteritis there is a significant rise in serum IgM levels. The rise seen in IgM following resection does not occur after bypass surgery. No consistent changes were noted in the concentrations of IgA or IgG. There was no observed difference noted between patients with ulcerative colitis and granulomatous enteritis in the IgM response following resection. The results resemble those which occur after the surgical removal of an allograft which is in process of being rejected.

There is increasing evidence that there may be some autoimmune basis for granulomatous and ulcerative disease of the bowel. However, the failure to demonstrate abnormalities in the serum levels of immunoglobulin has been disturbing. Recently some of the serological observations in these diseases have been attributed to decreased immune reactivity, the fall in gamma globulin with increased activity of disease and the rise with clinical improvement (De Dombal, 1967a). But these findings should be considered with other observations suggesting that the serum immunoglobulin concentration may not reflect the real magnitude of the immune response in these conditions. De Dombal (1967b) has reported an arteriovenous drop in gamma globulin across diseased segments of bowel in ulcerative colitis and immunofluorescent studies have demonstrated a perivascular accumulation of IgM in granulomatous disease of the bowel (Schofield, Deodhar, and Turnbull, 1969).

The striking simularity of these studies to that seen in the well known immune process of allograft rejection (Burrows, Gelernt, Ein, Dreiling, and Kark, 1964; Zühlke, Deodhar, Nakamoto, and Kolf, 1967) prompted us to investigate patients with granulomatous and ulcerative disease of the bowel for a phenomenon seen in rejecting renal grafts: the rise in immunoglobulin Mlevels noted following the removal

'Aided by a grant from the National Foundation for Ileitis and Colitis.

Received for publication 6 October 1971. of an immunologically destroyed transplant.

Clinical Materials and Methods

Immunoglobulin levels were measured by a radial diffusion technique (Melpar plates) immediately before and within 96 hours following surgery. The normal values for this method in our laboratory are for IgA $150-250 \mathrm{mg} \%$, for IgM $60-170 \mathrm{mg} \%$, and for IgG 700-1,500 mg\%. Four groups of patients were studied: group I consisted of 14 patients with granulomatous disease of the bowel having resections of the diseased segment; group II of six patients having resections of the colon for ulcerative colitis; group III of four patients with granulomatous disease of the bowel having a bypass of the diseased segment; and group IV of 16 patients (the control group), that is, patients having gastrointestinal resections for conditions other than granulomatous or ulcerative disease of the bowel (diverticulitis, carcinoma, peptic ulcer). Patients requiring transfusions were eliminated from the study if blood samples could not be obtained before the transfusion.

\section{Results}

In all 14 of the group I patients (Fig. 1) there was a significant increase in IgM levels following removal of the diseased segment. There was no correlation with the preoperative level. There was no significant change in IgA or IgG.

The group II patients (Fig. 2) demonstrated a 
Before operation After resection

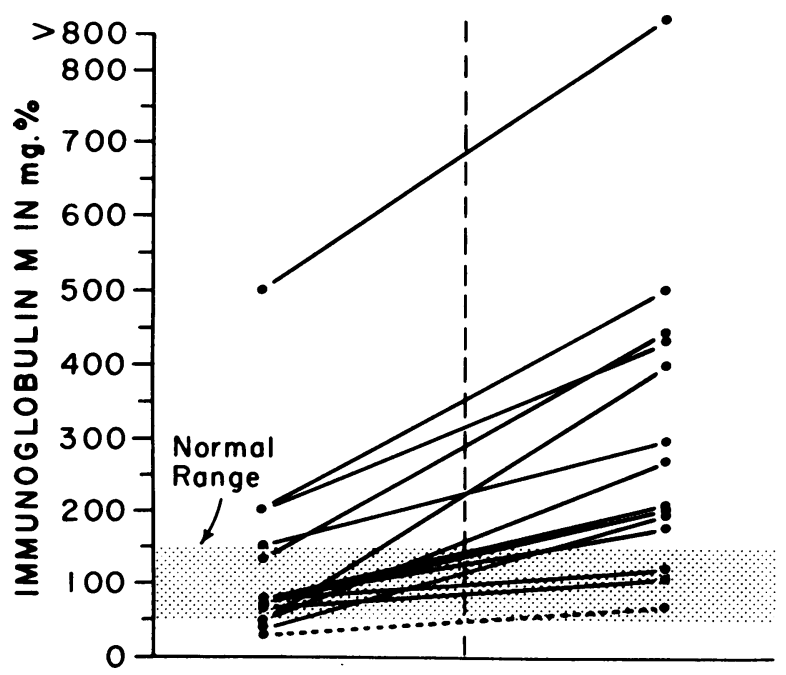

Fig. 1

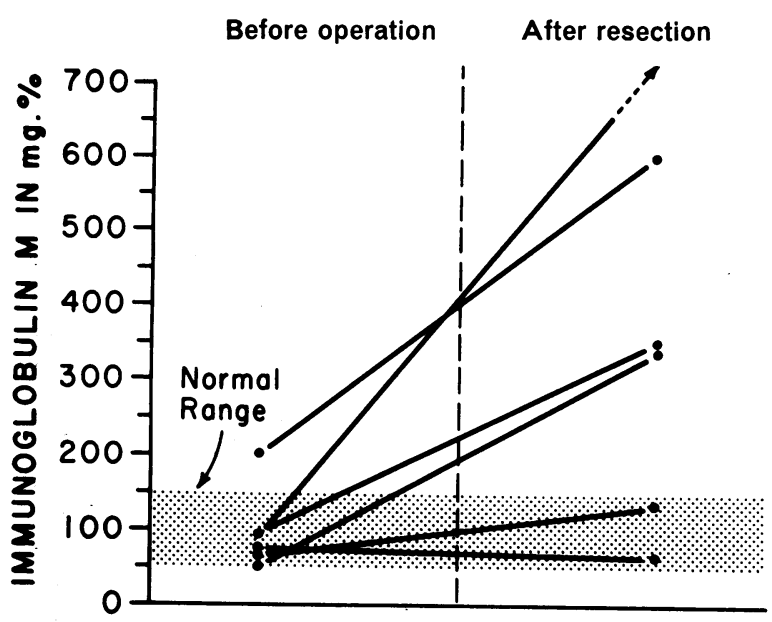

Fig. 2

similar rise in IgM following colectomy except for one patient who developed severe sepsis and was in shock for several hours after the dehiscence of an ileorectal anastomosis. As in the group I patients no significant changes were evident in IgA or IgG.

Group III (Fig. 3), four patients having a bypass with exclusion of the diseased segment of bowel, in contrast to the patients with resections, demonstrated no significant change in the IgM levels following surgery. There was no significant change in IgA or IgG levels.

Group IV, the control group of patients, demonstrated no alteration in immunoglobulin levels following resection.
Fig. 1 Serum immunoglobulin $M$ levels in patients with granulomatous disease of the bowel.

Fig. 2 Serum immunoglobulin $M$ levels in patients with ulcerative colitis.

Fig. 3 Serum immunoglobulin $M$ levels in patients with granulomatous disease of the bowel.

Before operation

After bypass

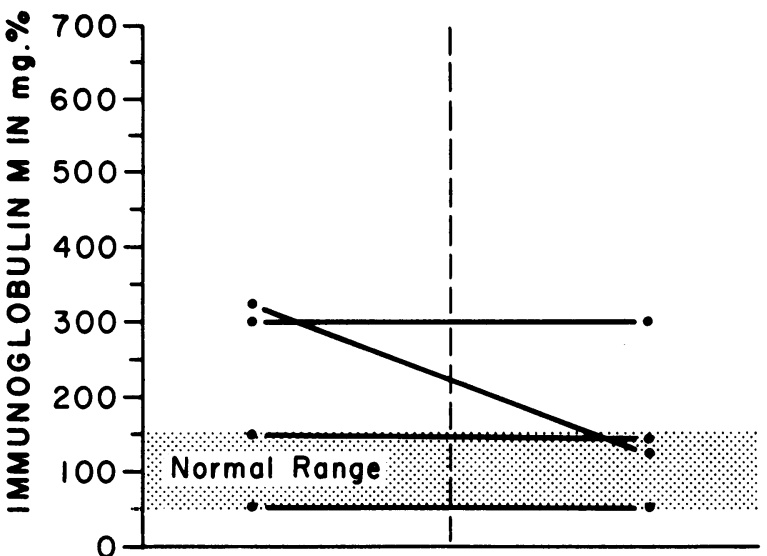

Fig. 3

\section{Discussion}

The consistent findings of the present study were the significant rise in IgM levels following resection of the bowel in both granulomatous and ulcerative colitis. No differentiation between the two conditions was apparent. The failure to demonstrate elevations of IgA, IgG, and albumin after resection (all three of lower molecular weight than IgM) makes it unlikely that leakage of IgM through the diseased segment was responsible for the increased levels noted after resection. In published studies with radioiodine-labelled IgM there was no preferential significant loss of IgM in the faeces (Jensen, Golter- 


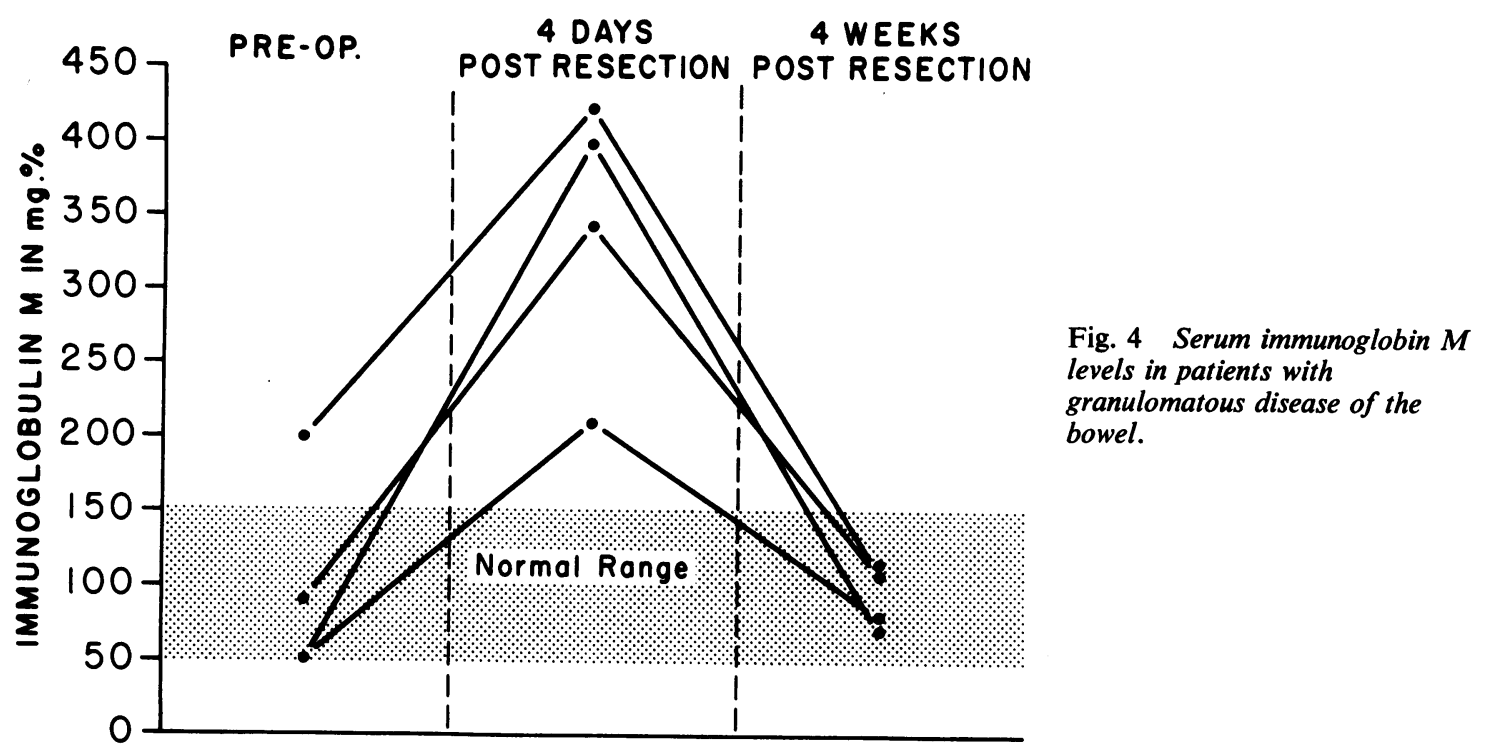

mann, Jarnum, Weeke, and Westergaard, 1970).

The failure to demonstrate elevation of IgM after bypass and the consistent rise after resection strongly suggests that there was binding of immunoglobulin $\mathbf{M}$ to the diseased bowel.

Preliminary studies in which IgM levels were measured one or more months following resection in four patients reveal a return approximately to preoperative levels (Fig. 4). These investigations suggest that there is significant binding of IgM to the bowel. The failure to see elevation of serum immunoglobulin levels during exacerbation of these diseases may be a reflection of binding and not of decreased production. It may well be that during increased activity of disease there is increased production of immunoglobulins and increased circulating levels are noted only when binding sites are removed. Then with perhaps an adjustment to a lower rate of production, possibly secondary to removal of antigenic stimuli, the concentration of IgM returns to lower levels.

The similarity of our results to the immune process of transplant rejection is readily apparent. However, it would be naive in our current state of knowledge to consider that our findings suggest that IgM is a causative factor in these disorders; rather it may be that these antibodies are a consequence or merely a concomitant of ulcerative and granulomatous disease of the bowel.

\section{References}

Burrows, L., Gelernt, I. M., Ein, S., Dreiling D. A., and Kark, A. E. (1964). Antigen antibody reactions involved in transplantation phenomena. Surg. Forum, 15, 135-137.

De Dombal, F.T.(1967a). The prognostic value of the serum proteins in ulcerative colitis. Brit. J. Surg., 54, 857-859.

De Dombal, F. T. (1967b). Serum proteins in ulcerative colitis: electrophoretic patterns in the inferior mesenteric artery and vein. Gut, 8, 482-485.

Jensen, K. B., Goltermann, N., Jarnum, S., Weeke, B., and Westergaard, H. (1970). IgM turnover in Crohn's disease. Gut, 11, 223-228.

Schofield, P. F., Deodhar, S. D., and Turnbull, R . B. (1969). Immunoglobulins in non-specific ulcerative disease of the colon. J. Roy. Coll. Surg. Edinb., 14, 157-160.

Zühlke, V., Deodhar, S. D., Nakamoto, S., and Kolff, W. J. (1967). Serum immunoglobulin levels following human renal transplantation: a preliminary report. Transplantation, 5, 135-141. 